\title{
béton compacté ou remblai rigidifié
}

\section{compacted concrete or stiffened embankment}

\author{
E. LEDEUIL \\ Ingénieur Arts et Métiers \\ Docteur en Mécanique des Sols \\ E.D.F. Région d'Équipement Alpes-Marseille*
}

\section{Résumé}

Les graves-ciment sont largement utilisés en France et ailleurs depuis des années surtout en travaux routiers, mais quelle est donc la différence capable de mobiliser tous les constructeurs et concepteurs de barrages depuis seulement quelques mois ou au maximum quelques années, lorsque l'on parle de "Béton Compacté n. C'est à cette question que l'auteur a essayé de répondre.

\footnotetext{
Abstract

The gravels-cement have been widely used in France and else where for many years particulary for road's works, but what is the difference capable to mobilize all dam constructors during the past few months or at most few years, when one speaks about "Roller Compacted Concrete". This is the question that the author has tried to answer.
} 


\section{INTRODUCTION}

Il n'y a même pas un an que cette nouvelle technique est apparue et elle a surpris de nombreux ingénieurs avec la deuxième partie de la question 57 du $15^{2}$ Congrès International des Grands Barrages, intitulée : * Béton Compacté au Rouleau ».

Un grand nombre d'ingénieurs se sont alors trouvés confrontés à cette nouvelle manière de concevoir et de construire des barrages. Un certain nombre de travaux avaient été effectuês avec cette technique mais ne possédaient ní nom ni publicité. Le tableau 1 donne une des premières listes établies souvent avec des appellations rétro-actives. On a pu ces derniers mois entendre parler de a Béton Compacté * (B.C. en français ou B.C.R. = Béton Compacté Roulé) ou de R.C.C. (en anglais Roller Compacted Concrete).

L'histoire suivante vécue par l'auteur est très significative.

En mai 1986, un Conseil étranger, chargé par E.D.F. de réaliser une analyse critique d'un projet de barrage en Guyane, avait terminé son rapport par une petite phrase :

* Une solution en * Béton Compacté * permettrait de réaliser une économie de $150 \mathrm{MF}$ sur le devis...» Immédiatement nous nous sommes lancés dans l'étude de cette nouvelle technique et de ses incidences.

La première idée qui nous fut suggérée fut de remplacer la digue derrière l'usine par du "Béton Compacté * pour gagner $105 \mathrm{MF}$ ! La réponse était évidemment négative car mettre quatre fois moins de matériau quatre fois plus cher garde le même coût.

Toutefois ayant obtenu Pautorisation de revoir entièrement la conception et l'organisation nous avons trouvé les moyens d'espérer de sérieuses économies.
Remarquant la rapidité d'exécution de cette nouvelle technique, le chemin critique du projet pouvait ne plus passer par les barrages. L'usine seule devait être prise en compte; or il fallait trois ans pour faire une telle usine; le délai global serait de trois ans et les barrages pouvaient être réalisés en temps masqué pourvu que leur conception soit modifiée. On pouvait donc gagner un an et demi ou deux ans, gain très appréciable en pays lointain. Un deuxième avantage concernait la conception où des ouvrages beaucoup plus simples pouvaient être envisagés, bénéficiant en particulier des capacités de submersion des barrages en cours de construction. Les ouvrages provisoires tels les batardeaux pouvaient être simplifiés et devenir d'usage de très courte durée; ils pouvaient s'inclure dans des périodes d'étiage... On pourrait continuer à parler de cet ouvrage mais tel n'est pas le but de cet article.

Un deuxième exemple peut être décrit sur un site en France où le maître d'ouvrage ne voulait pas d'un mur en béton de 50 mètres de haut, même en «Béton compacté», pour remplacer la digue en terre prévue en premier projet. L'auteur a alors proposé de donner à ce matériau l'appellation nouvelle, plus que de réalité nouvelle, de « REMBLAI RIGIDIFIE „ qui en fait explique l'origine de cette nouvelle technique. Il s'agit en effet de rigidifier un remblai, qui normalement s'étale avec des pentes amont et aval de l'ordre de 2 à 3 pour 1 , pour le ramener à des raideurs plus grandes que 1 pour 1 , soit 0,8 ou 0,7 ou même 0,6 pour certains projets américains. Quant à l'amont, avec un coffrage grimpant ou d'autres techniques, la verticale permet d'économiser presque la moitié inutile du barrage. Le résultat est une masse de remblais 4 à 5 fois plus faible (fig. 1).

La nécessité d'aller vite pour limiter l'effet des reprises oblige à concevoir un jeu de pistes évolutives dès l'origine. Ces pistes peuvent alors être conservées et orga-

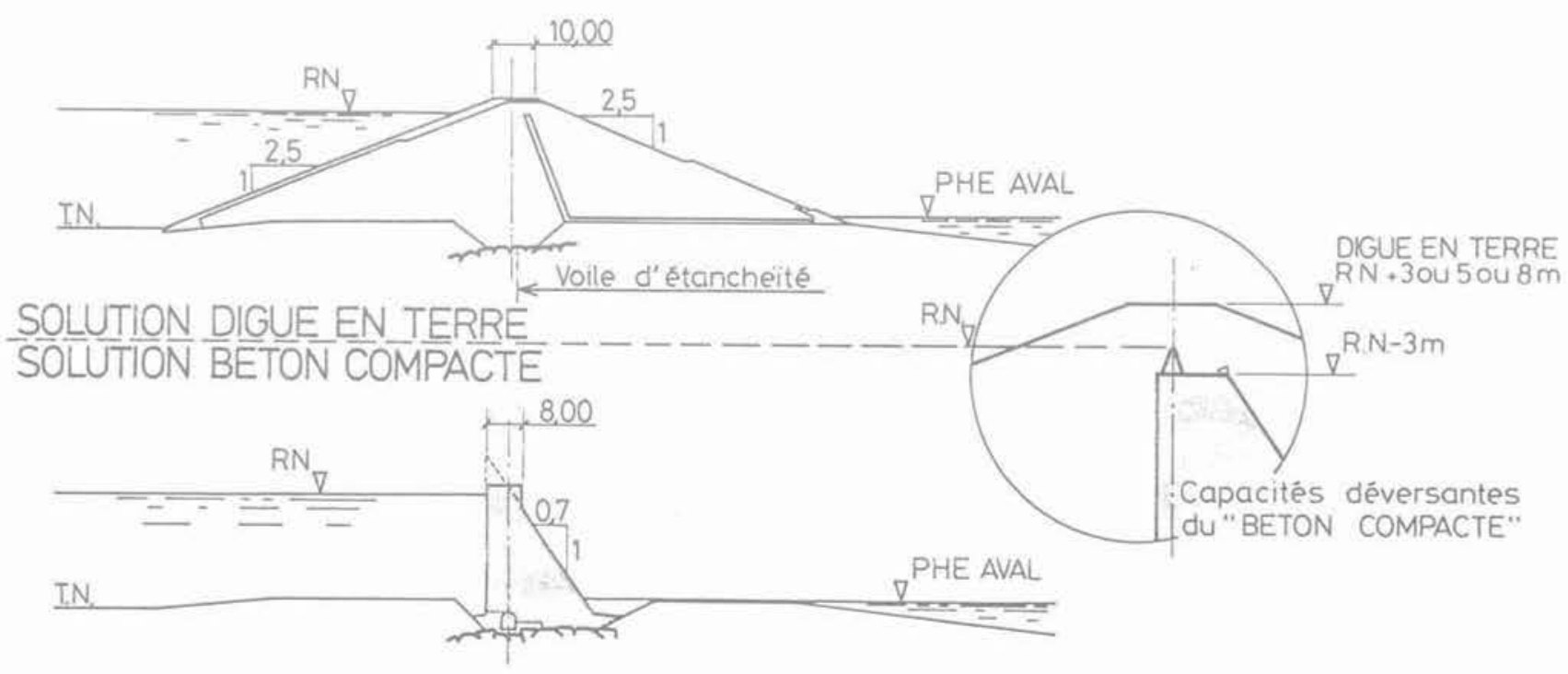

Fig. 1. - Etude comparative d'une solution "TERRE» et d'une solution «BÉTON COMPACTÉ». 
Tableau I. - Principaux ouvrages réalisés ou prévus en béton compacté (liste écourtée).

\begin{tabular}{|c|c|c|c|}
\hline Nom & Pays & Année & Volume $\mathrm{m}^{3}$ \\
\hline 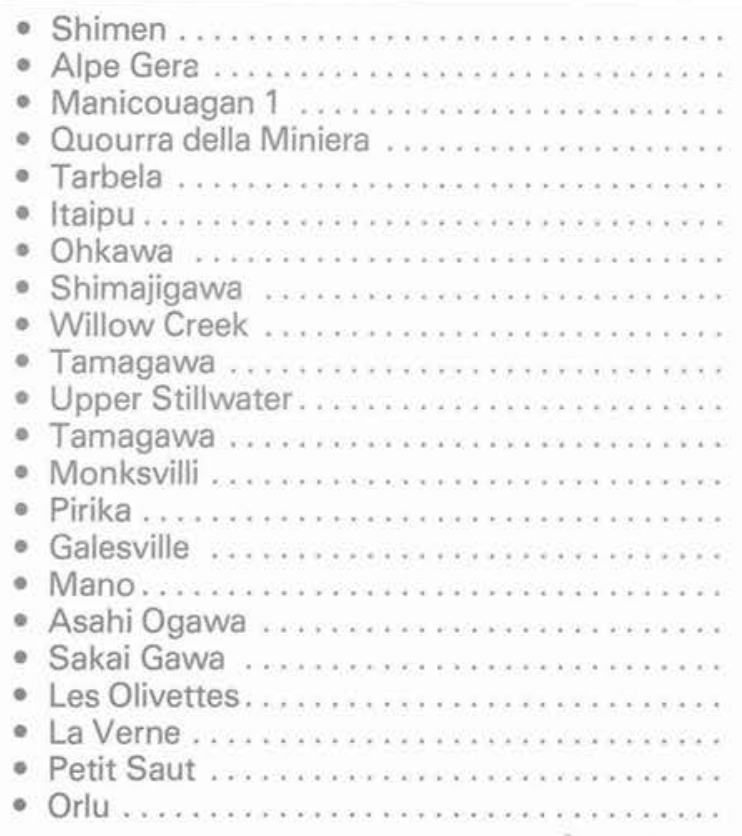 & $\begin{array}{l}\text { TW } \\
\text { IT } \\
\text { CD } \\
\text { IT } \\
\text { PR } \\
\text { PR/BZ } \\
\text { JP } \\
\text { JP } \\
\text { US } \\
\text { JP } \\
\text { US } \\
\text { JP } \\
\text { US } \\
\text { JP } \\
\text { US } \\
\text { JP } \\
\text { JP } \\
\text { JP } \\
\text { F } \\
\text { F } \\
\text { F } \\
\text { F }\end{array}$ & $\begin{array}{c}1960 \\
1964 \\
1965 \\
1968 \\
1974 / 8 \\
1978 \\
1979 \\
1981 \\
1983 \\
1984 \\
1985 \\
1988 \\
1986 \\
P \\
P \\
P \\
P \\
P \\
1986 / 7 \\
P \\
P \\
P\end{array}$ & $\begin{array}{r}1700000 \\
20000 \\
650000 \\
2800000 \\
26000 \\
300000 \\
165000 \\
329000 \\
750000 \\
963000 \\
1000000 \\
230000 \\
120000 \\
166000 \\
106000 \\
174000 \\
310000 \\
76000 \\
200000 \\
210000 \\
650000\end{array}$ \\
\hline
\end{tabular}

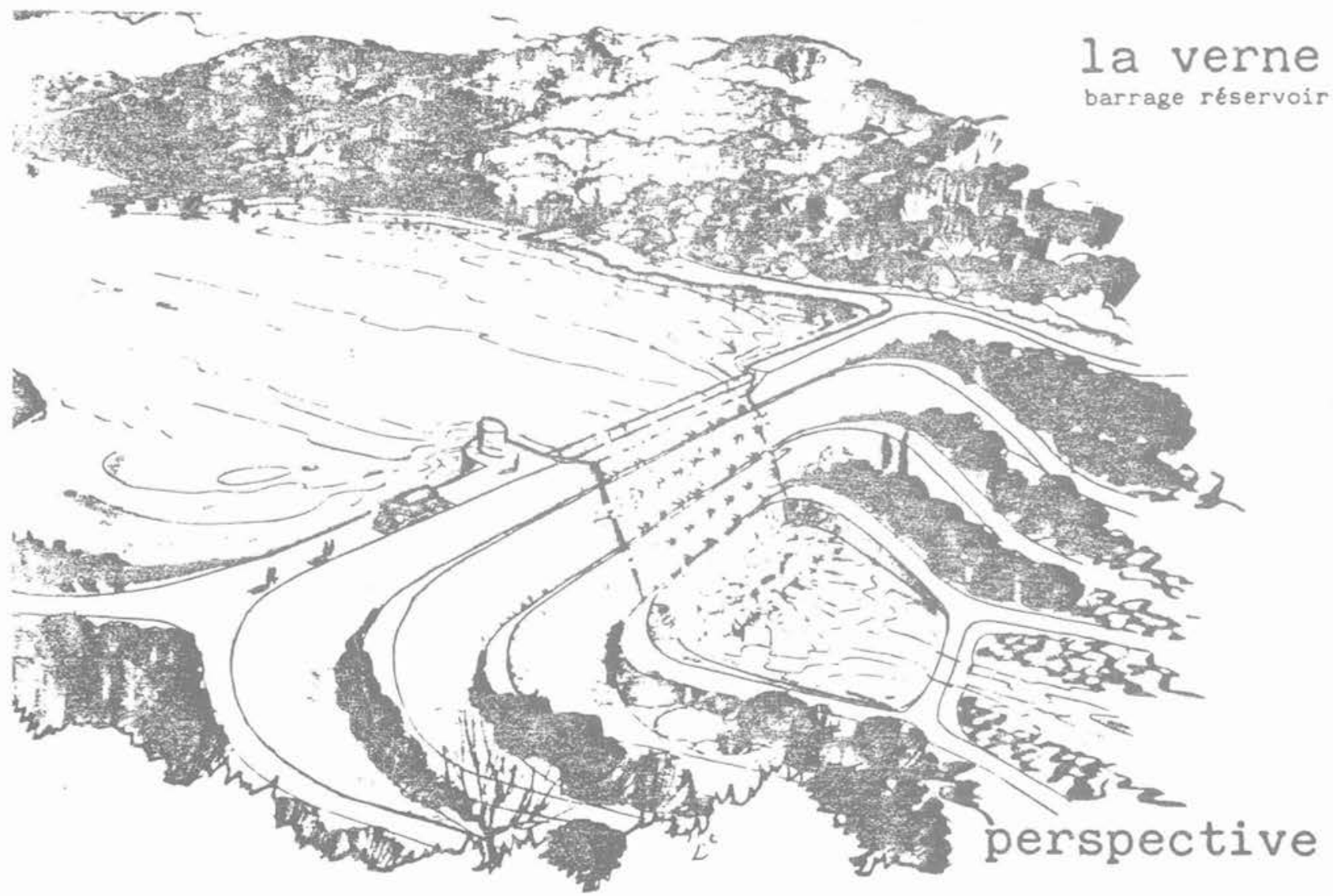

Fig. 2. - Etude paysagère. 


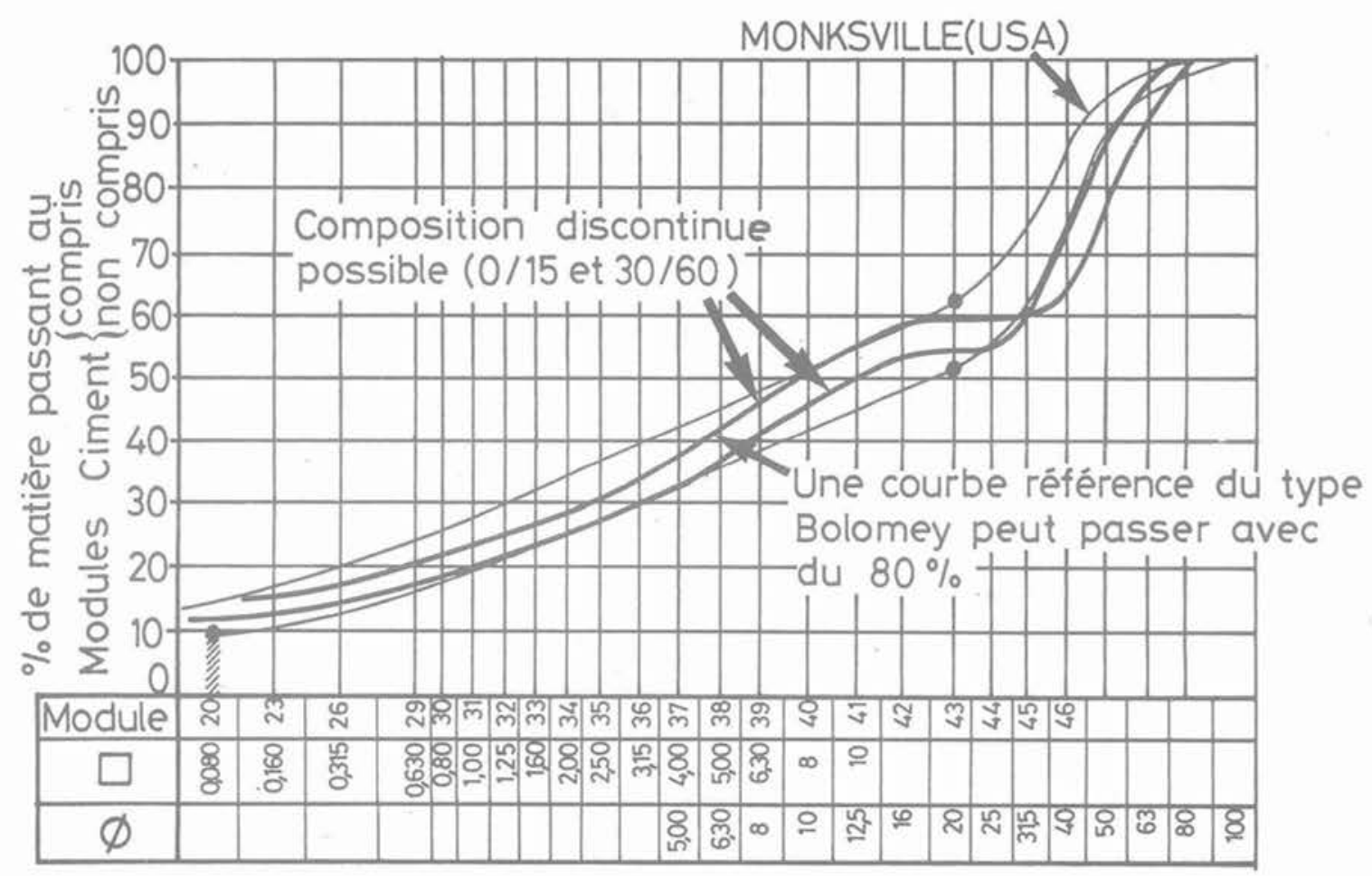

Fig. 3. - Composition prévue à Monksville (U.S.A.) et application à Petit Saut en discontinu.

nisées afin de supprimer l'effet de mur en béton. L'insertion dans le paysage peut alors y gagner (fig. 2).

Nous parlons de remblai, ... parce qu'il s'agit effectivement de remblai. La mise en place se fait avec des engins de terrassement comme tout remblai. Il n'y a plus de plots en béton, avec des joints, tout est traité et doit être traité en continu. Bien sûr, tout n'est pas aussi simple que cela mais la technique est lancée et a de sérieuses chances de réussir.

\section{QU'EST-CE QUE LE BÉTON COMPACTÉ?}

Il s'agit d'un béton, c'est-à-dire d'un assemblage de cailloux sélectionnés capables de se serrer au maximum (courbes de références de FAURY ou BOLOMEY (fig. 3) auquel on ajoute un liant, en principe du ciment et un peu d'eau. On doit alors utiliser un malaxeur à grand débit du type centrale routière à axe horizontal et travaillant en continu. Suivant les chantiers il faut des capacités de production de $600 \mathrm{t} / \mathrm{h}$ à $1000 \mathrm{t} / \mathrm{h}$. On remarquera que les centrales de $200 \mathrm{t} / \mathrm{h}$ parfois suffisantes pour de petits chantiers ne permettent pas l'utilisation de cailloux supérieurs à 30 ou $40 \mathrm{~mm}$ ce qui est regrettable.

Voilà des années que l'on fait des graves-ciments sur les routes ( 2 millions de $\mathrm{m}^{3}$ par an en graves ou sableslaitier, ciment ou chaux...) mais jamais ce matériau n'a eu l'honneur de devenir noble au point de faire des barrages.

S'agissant d'un remblai, de par sa mise en place, il est logique de retrouver les courbes de compactage de
«PROCTOR». Comme on le voit sur un graphique type (fig. 4), on peut espérer serrer les matériaux à des compacités plus élevées que celles normalement obtenues pour les bétons. Ceci a plusieurs conséquences:

Pour obtenir des serrages à des densités de 2,5 avec des agrégats dont la densité est de 2,7 , la teneur en eau doit impérativement être inférieure à $3 \%$ ce qui devient très vite une limite. En effet, il faut mouiller les cailloux, soit entre 1 et $2 \%$, et il faut hydrater le ciment. Il faut surveiller l'évaporation mais elle est limitée par la rapidité d'exécution.

Comme les densités sèches sont très fortes grâce au compactage puissant, l'étanchéité est améliorée car il y a moins de place pour l'eau. Mais alors le problème de l'étanchéité et de la porosité se pose car si l'eau est trop pure ou trop acide la percolation peut détruire l'assemblage interne des liants. Deux écoles s'affrontent donc. L'une trouve ce matériau digne d'un béton suffisamment étanche et n'utilise qu'une amélioration de parement par un sur-dosage en liant (par exemple $80 \mathrm{~kg}$ dans la masse et $150 \mathrm{~kg}$ par $\mathrm{m}^{3}$ en parement). L'épaisseur de la couche de surface ainsi renforcée en liant doit être faible pour ne pas faire apparaitre le phénomène de fissuration du béton. La deuxième école, pour l'instant la plus nombreuse dit qu'il vaut mieux une bonne étanchéité associée à un drainage sérieux, permettant d'être moins strict sur la qualité du béton de masse (voir le profil type de la figure 5). Plusieurs solutions du problème du masque-amont sont en cours de mise au point.

Ce sont les Japonais qui ont repris en les soignant, l'usage de ces matériaux rustiques. Ils ont presque réalisé du béton traditionnel, la mise en place est plus 


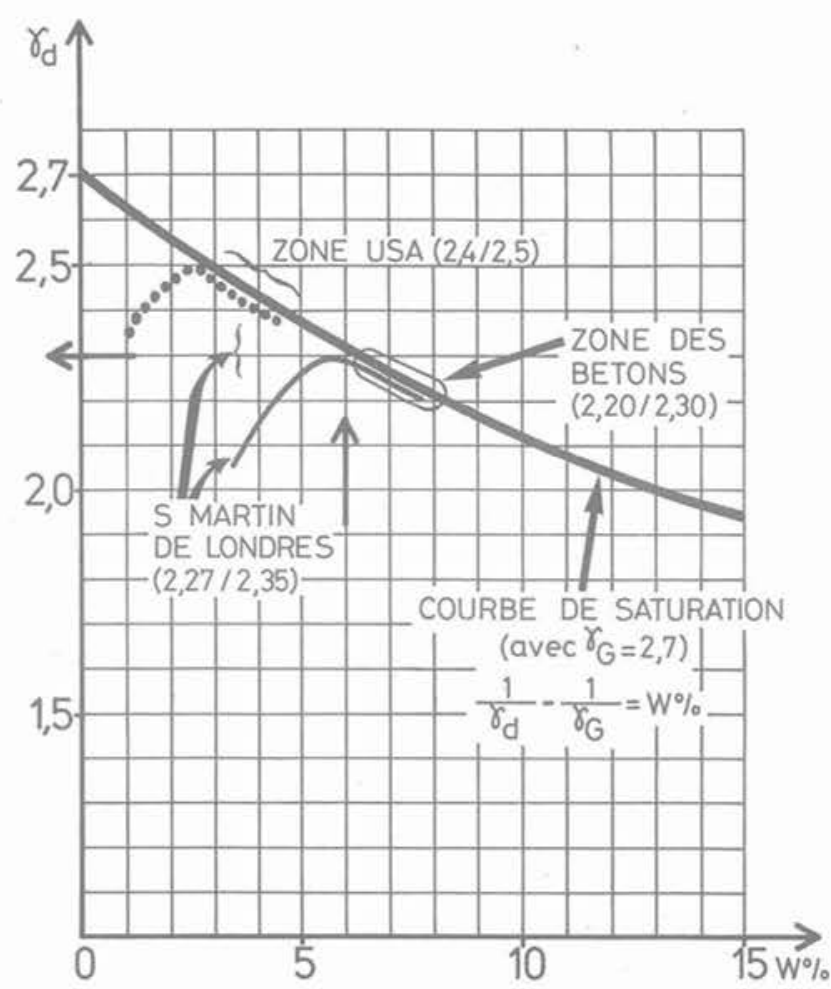

Fig. 4. - Densités sèches comparées sur matériaux frais.

rapide, mais le dosage en liant reste fort et pour limiter les effets du retrait alors inévitable (forts dosages donc mortier plus abondant) ils scient les joints.

Les Américains, eux, ont voulu trouver des solutions plus économiques pour des ouvrages écréteurs de crues donc toujours vides ou presque. Leur technique est alors très rapide, l'étanchéité est médiocre d'où des reprises coûteuses sur Willow Creek laissant quand même un bilan très favorable.

\section{COMPOSITION DU BÉTON COMPACTÉ}

La courbe de la figure 3 montre qu'il faut un minimum de $10 \%$ d'éléments inférieurs à 80 microns mais pas beaucoup plus. D'autre part il faut un grain maximum de $80 \mathrm{~mm}$, en effet les plus gros cailloux ont tendance à favoriser la ségrégation. Les plus petits coûtent cher puisque l'on peut introduire près de $50 \%$ de cailloux $40 / 80 \mathrm{~mm}$, cela valorise le béton de base $0 / 25 \mathrm{~mm}$ par exemple.

Si l'on regarde la courbe de la figure 6 , elle montre un béton trop riche en sable grossier qui sur place a rendu le béton plus souple n'atteignant pas facilement le blocage. Cet écart est obtenu par comparaison à des compositions type de FAURY ou BOLOMEY dites à remplissage maximal.

Le dosage en liant peut, dans la masse, être de $80 \mathrm{~kg} / \mathrm{m}^{3}$... mais il peut être moins fort et même inférieur à $50 \mathrm{~kg}$ avec un complément de $30 \mathrm{~kg}$ de cendres volantes.

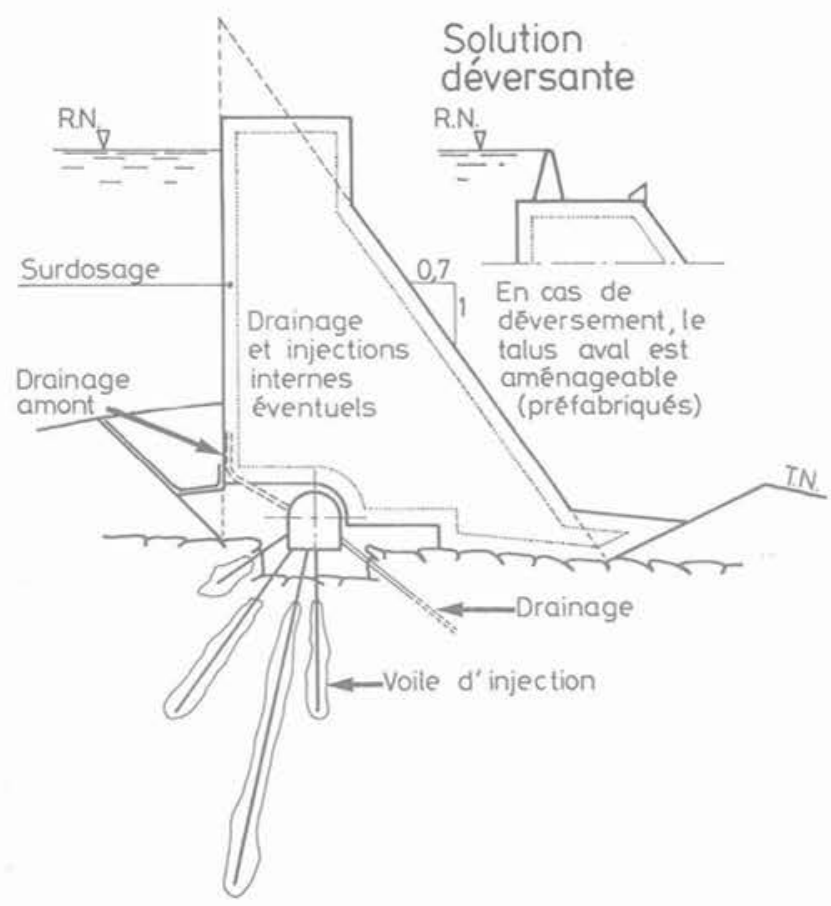

Fig. 5. - Profil type "BÉTON COMPACTÉ».

Pour l'entreprise BEC à Saint-Martin de Londres, un mélange de cendres et de laitiers sous le nom de ROLAC DE LAFARGE a permis avec un prix de revient moindre d'augmenter le dosage à $135 \mathrm{~kg}$ en apportant par ce biais les fines utiles. Ce mélange donne aussi une prise très lente et de très faibles montées en température. Il est un fait que dans cet exemple la centrale à béton de $200 \mathrm{t} / \mathrm{h}$ utilisée à Saint-Martin de Londres ne permettait d'admettre que des cailloux de $31,5 \mathrm{~mm}$ et qu'un ajout de gros cailloux aurait baissé sensiblement le dosage. La température de prise du béton à Saint-Martin de Londres après s'être élevée de $10^{\circ} \mathrm{C}$ a commencé à redescendre après 8 jours.

L'épaisseur des couches est souvent de l'ordre de 30 à $35 \mathrm{~cm}$. Il s'agit d'une épaisseur facile à répandre et à régler pour des remblais. Le compactage se fait par un ou deux passages sans vibration pour tasser et répartir, puis avec vibration. Certains ingénieurs prévoient aussi la mise en place par couches de l'ordre de $30 \mathrm{~cm}$, mais avec un compactage toutes les trois couches soit $90 \mathrm{~cm}$, ceci dans le but de limiter l'effet des reprises sans nuire au serrage optimal en utilisant des engins lourds et performants. Des essais doivent toujours être faits avant travaux afin de sélectionner les engins les mieux adaptés.

\section{CONCLUSIONS}

Il est certain que cette technique est appelée à se développer. On a même pu dire que $60 \%$ des barrages effectués au cours des quinze dernières années auraient pu, ou même auraient dû, être exécutés en Béton Compacté. 


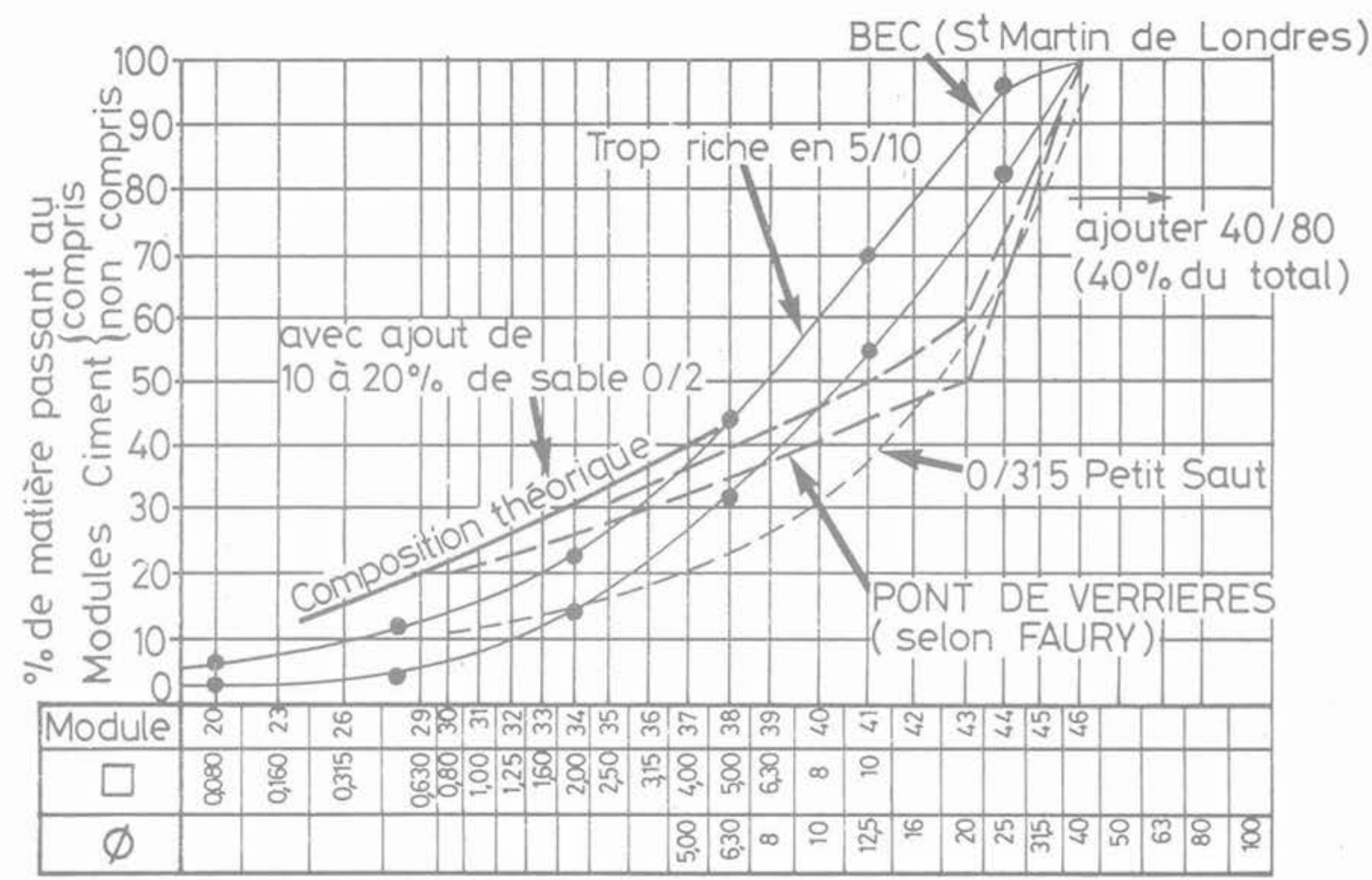

Fig. 6. - Comparaison des compositions en continu de Pont de Verrières et de Saint-Martin de Londres.

Entre les Japonais (jugés conservateurs dans cette technique) et les Américains (jugés plutôt novateurs) des excès dans les deux sens permettent de situer entre les deux les solutions de demain :

- Il faut limiter le mortier et l'eau.

- Il faut serrer au maximum les cailloux (s'ils sont en contact il n'y aura pas de retrait).

- Il faut limiter ou éviter les problèmes de reprise, c'est-à-dire monter le remblai en continu (il ne doit plus y avoir de dimanche sur le chantier...) à raison d'environ $1 \mathrm{~m} /$ jour et utiliser des liants à prise lente et donc peu exothermiques.

- L'épaisseur normale des couches est de 30 à $35 \mathrm{~cm}$ à la mise en place. Le compactage est à mettre au point par digue d'essai.

- Il faut limiter les risques de ségrégation en utilisant une dimension maximale de cailloux de $80 \mathrm{~mm}$.
Il semble intéressant d'utiliser des matériaux concassés capables d'être mis en place par vibration lourde (500 N par centimètre de génératrice) et de s'imbriquer puis de s'immobiliser ce qui est intéressant pour le compactage de couches ultérieures sur béton de souscouche frais. Les essais effectués à Pont de Verrières prouvent qu'au-delà de quelques passages le remblai se bloque.

Au niveau du prix de revient, le chantier doit être simplifié et le délai est nécessairement plus court. Le prix synthétique d'un Barrage en Béton Compacté y compris les parties en béton peu nombreuses ne doit pas dépasser $400 \mathrm{~F}$ en France avec des volumes suffisants (plus de $100000 \mathrm{~m}^{3}$ ) et une carrière facile.

Enfin, on peut souligner à nouveau les économies importantes que l'on peut espérer en utilisant, à la conception, les capacités de submersion des ouvrages en «BETON COMPACTÉ » ou en a REMBLAI RIGIDIFIÉ „ étanché et drainé à l'amont. 